\title{
Kaposi's sarcoma, a South African perspective: Demographic and pathological features
}

\author{
R D Mohanlal, MB ChB, FCPath (SA) Anat, MMed Anat Path; S Pather, MB BCh, FCPath (SA) Anat, MMed Anat Path \\ Department of Anatomical Pathology, Faculty of Health Sciences, University of the Witwatersrand and National Health Laboratory Service, \\ Chris Hani Baragwanath Academic Hospital, Johannesburg, South Africa
}

Corresponding author: R Mohanlal (reena.mohanlal@nhls.ac.za)

\begin{abstract}
Background. The incidence of Kaposi's sarcoma (KS) has increased dramatically since the onset of the AIDS epidemic. Of the estimated 66200 cases of KS worldwide, 58800 are considered to have occurred in sub-Saharan Africa

Objectives. To describe the epidemiology and pathological characteristics of KS at Chris Hani Baragwanath Academic Hospital (CHBAH), Johannesburg, South Africa.

Methods. A retrospective cross-sectional study design was used. Nine hundred and thirty-eight histopathology reports of KS diagnosed in 901 patients at CHBAH between 2005 and 2009 were reviewed. Age, gender, topographic site, CD4 count, HIV status, KS histological stage, findings of human herpesvirus 8 latency-associated nuclear antigen 1 immunohistochemistry and concomitant pathological findings were recorded.

Results. The male/female ratio was 1.2:1, the mean age 37 years and the median CD4 count 128 cells/ $\mu \mathrm{L}$. Lower limb skin biopsies accounted for $49.6 \%$ of cases. Paediatric, visceral and endemic KS accounted for only limited proportions (1.4\%, $1.4 \%$ and $1.3 \%$ of biopsies, respectively). There were concomitant pathological findings in $4.6 \%$ of biopsy specimens, infections and inflammatory dermatoses being the most frequent. Conclusion. The findings of this study highlight the need for allocation of diagnostic and treatment resources for KS. Documentation of the various demographic aspects of KS will prove to be of historical, clinical and histopathological interest as the long-term outcomes of antiretroviral therapy begin to emerge.
\end{abstract}

S Afr Med J 2015;105(5):375-378. DOI:10.7196/SAMJ.8773

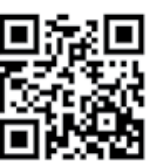

Kaposis sarcoma (KS) was first described by Moritz Kaposi in 1872. Four forms exist - classic KS, African/ endemic KS, AIDS-related KS and transplant-related KS. ${ }^{[1]}$ Of the estimated 66200 cases of KS worldwide in 2002, 58800 were considered to have occurred in sub-Saharan Africa (SSA). ${ }^{[2]}$

Although regarded as a neoplasm, KS is not a typical cancer. It is typically multifocal, it may regress with immune restoration, and the lesional cells are dependent on exogenous growth signals in vitro. ${ }^{[1]}$ Human herpesvirus 8 (HHV8) has been linked to KS. KS occurs most frequently in mucocutaneous sites. However, lymph nodes, visceral organs and unusual sites such as bone may also be involved. ${ }^{[3]}$ Patients may present with minimal or disseminated disease. Clinically, early skin and mucosal lesions may be difficult to recognise as they appear as faint, red-brown to violet macules. The established lesions of KS present as violaceous papules, plaques or nodules. Patients who have bronchopulmonary KS usually present with respiratory symptoms such as cough and breathlessness, while patients with gastrointestinal tract (GIT) KS may present with abdominal pain, nausea, weight loss, GIT bleeding, intestinal obstruction and/or diarrhoea. ${ }^{[4]}$
KS is confirmed by histopathological examination of biopsy specimens and with relevant ancillary investigations such as HHV8 latency-associated nuclear antigen 1 (LNA-1) immunohistochemistry (IHC). The typical histopathological features encompass a spindle cell proliferation with formation of slit-like vascular spaces, extravasated red bood cells (Fig. 1), haemosiderin pigment, plasma cells and hyaline globules. Three histological stages, patch, plaque and nodular, are recognised based on the clinical appearance and increasing degree of spindle cell proliferation. Furthermore, special morphological variants of KS have been described, e.g. lymphangiomatous,

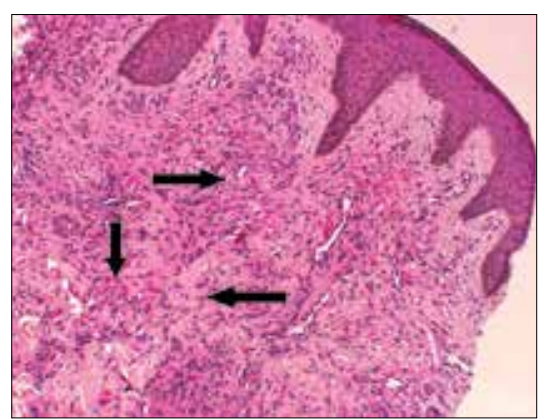

Fig. 1. Plaque-stage KS $(H \hookleftarrow E \times 200)$ showing spindle cell proliferation (left arrow) with slit-like vascular spaces (right arrow) and extravasated red blood cells (down arrow). lymphangiectatic, telangiectatic and keloidal variants among others. ${ }^{[5]}$

Despite the huge burden of disease in resource-limited settings, application of appropriate treatment options is possible and effective. Treatment options for AIDS-related KS include antiretroviral therapy (ART), local treatment, cytotoxic chemotherapy and targeted agents. ${ }^{[6]}$

The disease burden of KS in SSA is enormous. It is diagnosed on a daily basis at Chris Hani Baragwanath Academic Hospital (CHBAH), Johannesburg, South Africa (SA), yet published data from this hospital are lacking. The purpose of this study is to address some of the deficiencies in the literature as it pertains to patients at $\mathrm{CHBAH}$, the largest hospital in the southern hemisphere.

\section{Methods}

An observational retrospective crosssectional study design was used. All cases of KS diagnosed at the Histopathology Laboratory, Division of Anatomical Pathology, National Health Laboratory Service, CHBAH, from 1 January 2005 to 31 December 2009 were included by performing a SNOMED search of the DISA database using the code M-91403. Data were extracted from the histopathology and virology laboratory reports and 
entered onto a datasheet. The recorded variables included age, gender, HIV status (seropositive or seronegative), CD4 count (recorded when tested within 1 month of the confirmatory biopsy), topographic region biopsied, histological stage of $\mathrm{KS}$ (patch, plaque or nodular), HHV8 LNA-1 IHC and concomitant pathological features identified in the same biopsy specimen. Cases of paediatric KS were extrapolated from the main dataset. For the purposes of the study, 'paediatric' was defined as children $<14$ years of age as per the admission regulations at CHBAH. Nodal KS cases were reviewed by a single pathologist (RDM).

All information collected was extrapolated onto an Excel spreadsheet and STATISTICA 12 was used for descriptive statistics. Ethical approval was obtained from the Human Research Ethics Committee of the University of the Witwatersrand, Johannesburg (clearance certificate number: M130533). There was no direct contact with patients, and only linked codes were used during statistical analysis.

\section{Results}

In total, 938 biopsies from 901 patients were recorded. The male/female ratio was 1.2:1 (488 males and 412 females). Gender was unknown/unspecified in one patient.

Seven hundred and thirty-one patients (81.1\% of the total number) were HIVseropositive and 9 were HIV-seronegative as confirmed by HIV enzyme-linked immunosorbent assay (ELISA). HIV status was unknown in 161 (17.9\%) cases. There were $396 \mathrm{HIV}$-seropositive males and 334 $\mathrm{HIV}$-seropositive females (male/female ratio 1.2:1).

The mean (standard deviation (SD)) age at biopsy diagnosis of $\mathrm{KS}$ for the entire cohort was 36.8 (10.2) years, and 36.4 (9.7) years in the confirmed HIV-seropositive subgroup. The mean age for confirmed HIVseropositive males and females was 38.1 (9.3) and 34.5 (9.7) years, respectively.

Age was unspecified, and therefore not recorded, in 12 patients.

The median CD4 count recorded within 1 month of confirmed KS was 127.5 (quartile range $(\mathrm{QR})$ 184.5) cells/ $\mu \mathrm{L}$ and the mean (SD) was 155.74 (143.58) cells/ $\mu \mathrm{L}(n=540)$. Three hundred and eighty-two patients had CD 4 counts $<200$ cells $/ \mu L, 127$ had counts between 200 and 400 cells $/ \mu \mathrm{L}$, and 27 had counts between 400 and 600 cells/ $\mu \mathrm{L}$. HIV-seropositive males had a median CD4 count of 130 (QR 127.7) cells/ $\mu \mathrm{L}$ and HIV-seropositive females had a median CD4 count of 120.5 (QR 181.0) cells/ $\mu \mathrm{L}$.
Topographic sites were clinically unspecified in 264 biopsies. The majority of biopsies (where site was specified) were from the skin $(81.5 \%)$. One case involved the breast and showed spindle cells infiltrating among terminal duct lobular units. HHV8 IHC performed on this case was positive. Thirteen cases of visceral KS were identified, predominantly within the GIT (Table 1). HHV8 LNA-1 IHC stains were applied to 289 biopsies, $93.8 \%$ of which were positive.

Thirteen cases of paediatric KS were identified from 7 males and 6 females (male/female ratio 1.2:1). The mean (SD)

\begin{tabular}{ll}
$\begin{array}{l}\text { Table 1. Distribution of topographic } \\
\text { sites involved by KS }\end{array}$ & $\boldsymbol{n}$ \\
\hline Visceral & \\
Oesophagus & 1 \\
Stomach & 6 \\
Small bowel & 2 \\
Colon & 1 \\
Anus & 1 \\
Lung & 2 \\
Total & 13
\end{tabular}

Oral cavity
Tonsil

Tongue 8

Palate

Oral cavity NOS 4

Gingiva 3

Alveolar

Lip

Total

Pharynx/larynx 7

Lymph node $\quad 27$

Skin

Abdomen/trunk/back $\quad 17$

Lower limbs

Upper limbs

Head and neck

Chest/breast

Genital area

Total

\section{Eyelid}

Conjunctiva

Eye NOS

Total

Breast

NOS $=$ not otherwise specified. age in the paediatric group was 6.5 (3.4) years. HIV status was unknown in 2 paediatric patients, was confirmed to be negative in 1 and was positive in 10. Six CD 4 counts were available, with a median of 217.0 (QR 204.0) cells/ $\mu \mathrm{L}$. Lymph nodes $(n=5)$ were the most frequently represented topographic site in this subgroup. Other sites included palate (1), small bowel (1), skin of upper limb (1) and lower limb (2), and foreskin (2).

Twelve biopsies of KS from 9 HIV ELISA-seronegative patients (1 child and 8 adults) were seen. There was no history of transplantation in these patients. The male/female ratio was $1.3: 1$, the mean (SD) age was 55.4 (19.7) years, and the commonest site biopsied was skin of the lower limb.

Stages of KS were recorded in the histopathology reports of 708 biopsies. One hundred and nine cases (15.4\%) were of patch stage, $380(53.7 \%)$ were of plaque stage and 219 (30.9\%) showed nodular-stage KS. Special morphological variants were recorded in 17 cases. Eleven lymphangiomatous (Fig. 2), 1 telangiectatic and 5 lymphangiectatic variants were documented. Special morphological variants were most frequently noted in lower-limb skin biopsy specimens (41.2\%).

Concomitant pathological features were recorded in $4.6 \%$ of all biopsies, with infections constituting $27.9 \%$ of the additional pathology noted (Table 2). Seven cases of granulomatous inflammation and KS were seen in the same biopsy specimen. Five were recorded in skin punch biopsies, 1 from a lymph node and an additional case from the breast. Periodic acidSchiff and Ziehl-Neelsen special stains performed on all 7 cases were negative for micro-organisms. Polymerase chain reaction (PCR) for Mycobacterium genus was positive in 1 case of cutaneous KS which had concomitant granulomatous inflammation. Furthermore, acid-fast bacilli were detected in the sputum of an additional patient who had synchronous cutaneous KS and granulomatous inflammation. The features in two skin punch biopsies raised the possibility of concomitant syphilis owing to the presence of a dense lymphoplasmacytic infiltrate. In one case, Warthin-Starry special stain to detect spirochaetes was negative and serological confirmation was advised. Three cases of concomitant candidiasis were recorded in oral cavity biopsies. There were 27 cases of nodal KS, 12 of which showed concomitant pathology. One of these showed granulomatous 


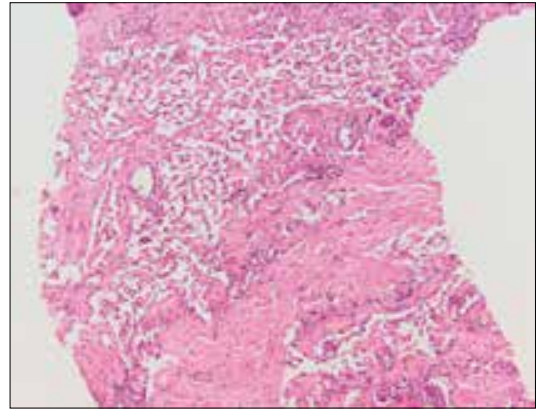

Fig. 2. Lymphangiomatous variant of $K S$ ( $H \bowtie E$ $\times 100$ ).

\begin{tabular}{|c|c|}
\hline Type of concomitant pathology & $n$ \\
\hline \multicolumn{2}{|l|}{ Infections } \\
\hline Cytomegalovirus & 3 \\
\hline Candida & 3 \\
\hline Cryptococcus & 1 \\
\hline Possible syphilis & 2 \\
\hline Herpes simplex virus & 1 \\
\hline $\begin{array}{l}\text { Granulomatous inflammation } \\
\text { due to Mycobacterium }\end{array}$ & 2 \\
\hline Subtotal & 12 \\
\hline \multicolumn{2}{|l|}{ Dermatitis } \\
\hline Seborrhoeic dermatitis & 1 \\
\hline Spongiotic dermatitis NOS & 1 \\
\hline Interface dermatitis & 4 \\
\hline $\begin{array}{l}\text { Granulomatous inflammation } \\
\text { NOS }\end{array}$ & 5 \\
\hline Subtotal & 11 \\
\hline Panniculitis & 4 \\
\hline \multicolumn{2}{|l|}{ Other } \\
\hline Human papillomavirus & 1 \\
\hline Verruca & 1 \\
\hline Chalazion & 1 \\
\hline Intussusception & 1 \\
\hline Gastritis & 1 \\
\hline HIV lymphadenitis & 10 \\
\hline Castleman's disease & 1 \\
\hline Total & 43 \\
\hline NOS $=$ not otherwise specified. & \\
\hline
\end{tabular}

inflammation (as above), 10 showed HIV lymphadenitis and the remaining case showed concomitant Castleman's disease.

\section{Discussion}

Of the tens of thousands of cases of KS diagnosed worldwide, the vast majority are believed to have occurred in SSA. ${ }^{[2]}$ While developed countries are experiencing a decline in the incidence of KS as a result of timeous ART initiation, KS remains a cause of significant morbidity and mortality in developing countries like SA owing to suboptimal availability of $\mathrm{ART}^{\left[{ }^{[7]}\right.}$

SSA is home to approximately $80 \%$ of the world's HIV-infected female population, and heterosexual transmission of HIV remains the main mode of spread worldwide. ${ }^{[8]}$ In $\mathrm{SA}$, the risk of developing $\mathrm{KS}$ is related to the transmission pattern of HIV, which is predominantly heterosexual. ${ }^{[9]}$ The male/ female ratio of patients diagnosed with confirmed HIV-associated KS in this study is in keeping with the trend of KS shifting from a male-predominant disease ${ }^{[10]}$ and is reflective of the high prevalence of HIV among young women, who bear the brunt of HIV in SA. This is in contrast to the initial recognition of KS as a male-predominant disease in homosexual men in the USA in the 1980s.

The mean age of patients diagnosed with $\mathrm{KS}$ at CHBAH was similar to the findings of Mosam et al. ${ }^{[9]}$ and may change as the longterm effects of the ART roll-out programme begin to emerge. It is predicted that with ART, HIV-seropositive patients will live longer and may therefore present later with KS. ${ }^{[11]}$

The mean and median CD4 counts we have demonstrated are lower than those documented in comparable studies. ${ }^{[9]}$ Interestingly, $28.5 \%$ of the 540 patients with known CD4 counts had values in excess of 200 cells $/ \mu \mathrm{L}$. This is in keeping with the suggestion that in Africa severe immunosuppression is not a prerequisite for the development of KS. ${ }^{[12]}$

KS most commonly involves mucocutaneous topographic regions. ${ }^{[3]}$ Our study is supportive of this, as at least $63.5 \%$ of all KS biopsies at CHBAH during the study period originated from mucocutaneous sites. The limited number of visceral KS may be attributed to under-sampling, as synchronous, more easily accessible mucocutaneous lesions in these patients may have been biopsied instead. KS involving topographic sites considered unusual by Pantanowitz and Dezube ${ }^{[13]}$ in this study included 2 cases in the larynx, 9 in conjunctiva and 1 in the breast.

HIV status was not available in $17.9 \%$ of patients. This may be attributed to a number of reasons, including patients refusing consent for HIV testing, HIV testing at a peripheral hospital not linked to the DISA database at $\mathrm{CHBAH}$, failure to recommend HIV testing or failure to specify the patients' status in the clinical history section of the laboratory requisition form.

Pitche et al. ${ }^{[14]}$ in a study of 20 cases of African/endemic KS, found a male/female ratio of 9:1 in contrast to the lower male/ female ratio of patients with endemic KS at CHBAH of 1.3:1.

Interestingly, HHV8 LNA-1 staining was only applied to $30.8 \%$ of biopsies at CHBAH during the study period. This reflects variable individual pathologists' practice, as in the appropriate clinical context the histopathological diagnosis may be based on the presence of typical morphological features of KS. In SA's public health sector, immunohistochemistry-related cost factors also play an influential role. In this study, the majority (93.8\%) of HHV8 LNA-1 immunohistochemical stains performed on KS biopsies were positive, more than the $64 \%$ positivity recorded by Ramos da Silva et al. ${ }^{[15]}$ HHV8 PCR testing may be indicated in those cases displaying clinical and morphological features of KS but negative HHV8 immunohistochemical staining. Paediatric KS (diagnosed in patients aged $<14$ years) accounted for $1.4 \%$ of all biopsies and lymph nodes $(n=5)$ were the most frequent site represented. The findings of Stefan et al. ${ }^{[16]}$ revealed a median age of 6 years, similar to our findings of a median of 7 years. However, their average CD4 count was higher (mean 440 cells $/ \mu \mathrm{L}$ v. 209.2 cells $/ \mu \mathrm{L}$ in our study), and the most commonly affected site was the skin. Further studies to determine the incidence of paediatric KS may be useful in light of the rigorous prevention of mother-tochild transmission antiretroviral programme, as the impact of reduced vertical transmission of HIV infection may result in a decline in the incidence of KS in children.

In this study, patch-stage KS was diagnosed least frequently of the three histological KS stages. Patch-stage KS may be a subtle manifestation clinically. It is probable that patients are more likely to recognise and be concerned about well-established and/ or larger lesions. Moreover, from a clinical viewpoint, larger lesions are likely to be more representative and provide greater diagnostic/confirmatory yield. Plaque- and nodular-stage KS lesions are therefore more frequently targeted for biopsy.

Granulomatous inflammation was seen in 7 of our specimens, with 2 cases of confirmed Mycobacterium infection. As highlighted by Ramdial et al., ${ }^{[17]}$ adequate investigation of granulomatous inflammation for confirmation of concomitant $M$. tuberculosis is crucial in highlighting possible multidrug resistance and non-compliance. The review article by Grayson ${ }^{[18]}$ addressed concomitant pathology in cutaneous KS. Our CHBAHbased study documents concomitant pathology occurring in extracutaneous KS biopsy specimens. As concomitant pathology was noted in approximately half of all 
KS-containing lymph node biopsy specimens, reporting pathologists should be vigilant.

Treatment options for AIDS-related KS include ART, local treatment (radiation, topical agents), cytotoxic chemotherapy and targeted agents. KS lesions regress with initiation of ART. ART inhibits HIV replication, decreases production of HIV-tat and ameliorates the immune response to HHV8 ${ }^{[6]}$ Semeere et al. ${ }^{[7]}$ demonstrated a reduction in the incidence of KS by $72 \%$ in ART users compared with non-ART users. These findings were corroborated by Bohlius et al., ${ }^{[19]}$ who concluded that the most effective treatment modality remains ART, as it significantly decreases morbidity and mortality. ART may be associated with immune reconstitution syndrome (IRIS). KS-IRIS has been well documented. Recently, it has been shown that the incidence of KS-IRIS is higher in Africa than in the UK, with $13.9 \%$ of individuals developing KS-IRIS, a rate 2.5 times higher than European cohorts. ${ }^{[20]}$ Chemotherapy which includes anthracyclines is used for treatment of visceral disease and progressive mucocutaneous lesions. Local therapy such as radiotherapy is useful for palliation, management of bulky lesions and cosmesis. In this era of molecular medicine, targeted pathogenesis-based treatments such as antiangiogenic compounds, retinoic acid, hormonal agents and antiherpes agents are also being used in the treatment of KS. ${ }^{[6]}$

\section{Study limitations}

A possible limitation of this study is that there was an element of sampling bias, as $\mathrm{CHBAH}$ is a tertiary hospital. Patients treated at this hospital are likely to have a greater burden of disease (i.e. lower CD4 counts and more advanced HIV infection) than those treated at local clinics. Clinical data regarding ART use were not available.

\section{Conclusion}

KS is a pathogenetically and morphologically diverse disease. The epidemiology has changed dramatically since AIDS-associated KS was first diagnosed in the 1980s. With the introduction of ART, the demographics may shift once again as the effects of therapy become apparent. In conclusion, this study highlights the clinicopathological aspects and burden of HIV-associated KS at $\mathrm{CHBAH}$ and includes limited representation of endemic, paediatric and visceral KS subgroups. The histopathological spectrum of KS, including special morphological variants, and presence of concomitant pathology have also been reported. The findings of this study serve as a foundation on which further comparative research could be based.
Acknowledgments. Special thanks to Prof. E Libhaber for assistance with statistical analysis, Susan Radebe for slide retrieval and Eric Liebenberg for assistance with photography. We acknowledge the contribution of patients and staff at $\mathrm{CHBAH}$ in making this institution an inspirational place to work at.

Funding acknowledgement. This research received no financial grants. SP was supported by SATBAT/FIC grant number 3U2RTW007370-05S1.

\section{References}

1. Ganem D. KSHV infection and the pathogenesis of Kaposi's sarcoma. Annu Rev Pathol 2006;1:273296. [http://dx.doi.org/10.1146/annurev.pathol.1.110304.100133]

2. Parkin D. The global health burden of infection associated cancers in the year 2002. Int J Cancer 2006;118(12):3030-3044. [http://dx.doi.org/10.1002/ijc.21731]

3. Radu O, Pantanowitz L. Kaposi sarcoma. Arch Pathol Lab Med 2013;137(2):289-294. [http://dx.doi. Radu O, Pantanowitz L. Kaposi
org/10.5858/arpa.2012-0101-RS]

Drg/10.5858/arpa.2012-0101-RS] Dezube B. Clinical presentation and natural history of AIDS related Kaposi's sarcoma. Hemat
Clin North Am 1996;10(5):1023-10229. [http://dx.doi.org/10.1016/S0889-8588(05)70382-8]

5. Grayson W, Pantanowitz L. Histological variants of cutaneous Kaposi sarcoma. Diagn Pathol 2008;3:31-39. [http://dx.doi.org/10.1186/1746-1596-3-31]

6. Cattelan A, Trevenzoli M, Aversa S. Recent advances in the treatment of AIDS-related Kaposis sarcoma. Am J Clin Dermatol 2002;3(7):451-462. [http://dx.doi.org/10.2165/00128071-200203070-00002]

7. Semeere A, Busakhala N, Martin J. Impact of antiretroviral therapy on the incidence of Kaposis sarcoma in resource rich and resource limited settings. Curr Opin Oncol 2012;24(5):522-530. [http:/ dx.doi.org/10.1097/CCO.0b013e328355e14b]

8. De Cock K, Jaffe H, Curran J. The evolving epidemic of HIV/AIDS. AIDS 2012; 26(10):1205-1213. [http://dx.doi.org/10.1097/QAD.0b013e328354622a]

9. Mosam A, Hurkchand H, Cassol E, et al. Characteristics of HIV-1-associated Kaposis's sarcoma among women and men in South Africa. Int J STD AIDS 2008;19(6):400-405. [http://dx.doi.org/10.1258/ijsa.2008.007301

10. Sitas F, Newton R. Kaposi's sarcoma in South Africa. J Natl Cancer Inst Monogr 2000;2000(28):1-4. [itas F, Newton R. Kaposi's sarcoma in South Africa. J Natl Cancer
[http://dx.doi.org/10.1093/oxfordjournals.jncimonographs.a024250]

11. Daly M, Fogo A, Mcdonald C, Morris-Jones R. Kaposi sarcoma: No longer an AIDS defining illness? Daly M, Fogo A, Mcdonald C, Morris-Jones R. Kaposi sarcoma: No longer an AIDS defining illness?
A retrospective study of Kaposi sarcoma cases with CD4 counts above $300 / \mathrm{mm}^{3}$ at presentation. Clin A retrospective study of Kaposi sarcoma cases with CD4 counts above
Exp Dermatol 2014;39(1):7-12. [http://dx.doi.org/10.1111/ced.12163]

12. Cassol E, Page T, Mosam A, et al. Therapeutic response of HIV-1 subtype C in African patients coinfected with either Mycobacterium tuberculosis or human herpes virus-8. J Infect Dis 2005;191(3):324-332. [http://dx.doi.org/10.1086/427337]

13. Pantanowitz L, Dezube B. Kaposi sarcoma in unusual locations. BMC Cancer 2008;8:190-198. [http:// dx.doi.org/10.1186/1471-2407-8-190]

14. Pitche P, Kombate K, Owono F, Tchangai-Walla K. Kaposi's sarcoma in a hospital setting in Lome (Togo): A study of 93 cases. Int J Dermatol 2007;46(Suppl 1):42-44. [http://dx.doi.org/10.1111/j.13654632.2007.03464.X]

15. Ramos da Silva S, Bacchi M, Bacchi C, Elgui de Oliviera E. Human bcl-2 expression, cleaved caspase-3 and KSHV LANA-1 in Kaposi sarcoma lesions. Am J Clin Pathol 2007;128(5):794-802. [http://dx.doi. org/10.1309/TFU2FXK3APOC9R2X]

16. Stefan D, Stones D, Wainwright L, Newton R. Kaposi sarcoma in South African children. Pediatr Blood Cancer 2011;56(3):392-396. [http://dx.doi.org/10.1002/pbc.22903] 17. Ramdial P, Sing Y, Subrayan S, et al. Granulomas in acquired immunodeficiency syndrome-associated
cutaneous Kaposi sarcoma: Evidence for a role for Mycobacterium tuberculosis. I Cutan Pathol cutaneous Kaposi sarcoma: Evidence for a role for Mycobacterium tubech
2010;37(8):827-834. [http://dx.doi.org/10.1111/j.1600-0560.2010.01544.x]

18. Grayson W. Recognition of dual or multiple pathology in skin biopsies from patients with HIV/AIDS Patholog Res Int 2011;2011:398-546. [http://dx.doi.org/10.4061/2011/398546]

19. Bohlius J, Valeri R, Maskew M, et al. Kaposi's sarcoma in HIV infected patients in South Africa: Multicohort study in the antiretroviral therapy era. Int J Cancer 2014;135(11):2644-2652. [http:// dx.doi.org/10.1002/ijc.28894

20. Letang E, Lewis J, Bower M, et al. Immune reconstitution inflammatory syndrome associated with Kaposi sarcoma: Higher incidence and mortality in Africa than in the UK. AIDS 2013;27(10):16031613. [http://dx.doi.org/10.1097/QAD.0b013e328360a5al]

Accepted 18 March 2015 